\title{
Valsalva Haemorrhagic Retinopathy
}

\author{
Balaji Kannan, Vijayalakshmi Balaji, Sherin Kummararaj, Govindarajan Kummararaj \\ $\operatorname{Dr}$ A Govindarajan Eye Hospital, Puthur, Trichy, India
}

\begin{abstract}
Valsalva haemorrhagic retinopathy is an uncommon clinical entity that can severely affect visual acuity. Valsalva haemorrhagic retinopathy is caused by a sudden increase in intrathoracic pressure, which reduces venous return to the heart and increases cephalic venous pressure, and may cause a sudden rise in intraocular venous pressure and spontaneous rupture of the retinal capillaries. This report is of a patient with a large macular pre-retinal haemorrhage that was managed conservatively.
\end{abstract}

Key words: Retina, Valsalva maneuver, Venous pressure

Asian J Ophthalmol. 2011;13:22-3.

\section{Introduction}

Valsalva haemorrhagic retinopathy is an uncommon clinical entity that can affect visual acuity to varying degrees, ranging from mild reduction to severe visual loss. ${ }^{1}$ Valsalva haemorrhagic retinopathy is caused by a sudden increase in intrathoracic pressure, which reduces venous return to the heart and increases cephalic venous pressure. This causes a sudden rise in intraocular venous pressure and spontaneous rupture of the retinal capillaries ensues. The venous pressure rise may also result in subconjuctival haemorrhages and skin petechiae. ${ }^{2}$ This report is of a patient with a large macular pre-retinal haemorrhage that was managed conservatively.

\section{Case Report}

A 44-year-old woman presented in 2009 with a 1-day history of sudden painless loss of vision in the left eye following an acute and severe episode of coughing. The patient had no previous history of ocular or other medical conditions. At presentation, the visual acuity in the right eye was 20/20 and in the left eye was counting fingers at $1 \mathrm{~m}$. Her intraocular pressure (IOP) was $19 \mathrm{~mm}$ $\mathrm{Hg}$ in both eyes. There was no relative afferent papillary defect. The anterior segment was normal in both eyes. Posterior segment examination revealed a large macular pre-retinal haemorrhage in the left eye, which was about 20 disc diameter in size, with focal haemorrhage along the superonasal arcades (Figure 1). Her blood pressure was 110/72 $\mathrm{mm} \mathrm{Hg}$. Her full blood count, blood glucose, and coagulation screen (prothrombin time) were normal. She was advised to avoid any strenuous exercise and to attend for review after 6 weeks.

\footnotetext{
Correspondence: Dr Balaji Kannan, 180/72 East Chitra Street, Srirangam Trichy, Tamil Nadu 620006, India.

Figure 1. Left fundus showing a pre-retinal haemorrhage of about 20 disc diameter overlying the macula (arrow) and a small isolated haemorrhage along the superonasal arcades (arrowhead).

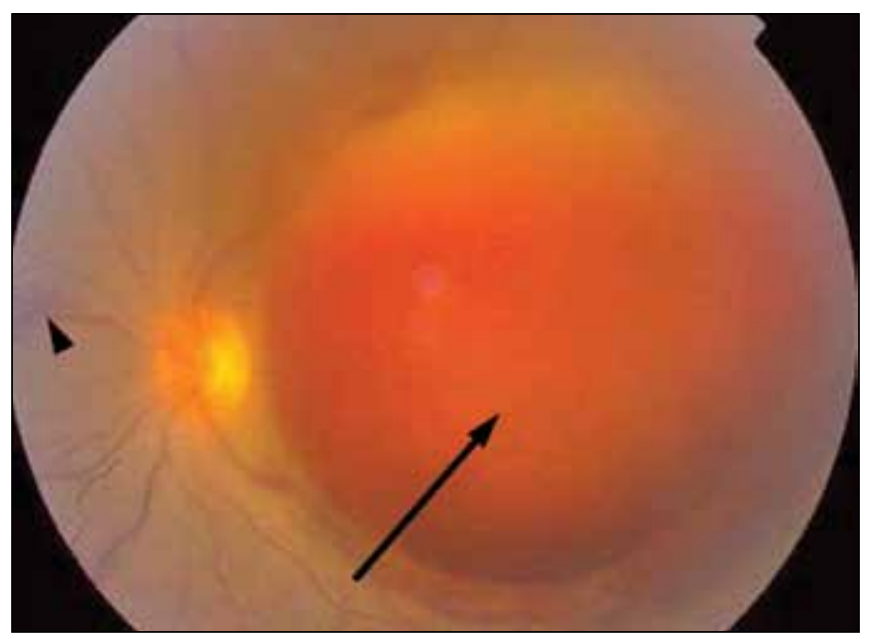

After 6 weeks, the visual acuity in the left eye was improved to counting fingers at $4 \mathrm{~m}$ and her IOP was normal. The left fundus showed partial resolution of the haemorrhage (Figure 2) and conservative management was continued. After 2 months, the visual acuity in the left eye had improved to $20 / 80$. The visual acuity continued to improve and returned to 20/20 unaided 7 months after the initial presentation. The left fundus showed resolution of the pre-retinal haemorrhage, but retinal pigment epithelial changes were noted (Figure 3). The patient was extremely pleased with her visual recovery without surgical intervention.

\section{Discussion}

Valsalva haemorrhagic retinopathy was first described by Thomas Duane in 1972, when this author reported 3 patients with macular pre-retinal haemorrhages secondary to sudden increase in intrathoracic pressure. ${ }^{2}$ Valsalva haemorrhagic retinopathy is 
Figure 2. Left fundus showing partial resolution of the pre-retinal macular haemorrhage after 6 weeks.

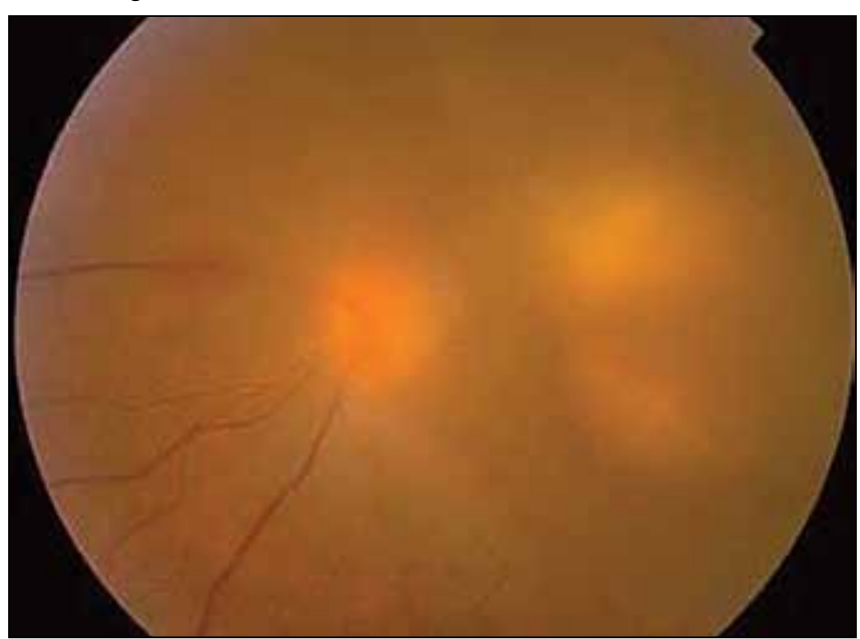

Figure 3. Left fundus showing complete resolution of the pre-retinal haemorrhage after 7 months. Retinal pigment epithelium changes can be seen (arrow).

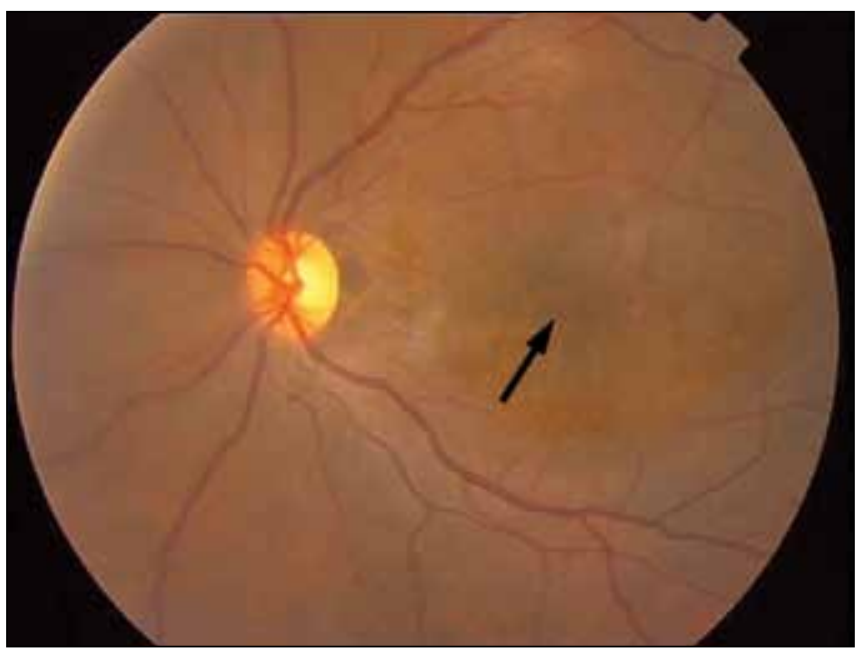

related to the Valsalva manoeuvre, named after the 17th century anatomist. Valsalva haemorrhagic retinopathy has been reported following aerobic exercises,${ }^{3}$ inflating balloons, ${ }^{4}$ constipation, vigorous sexual activity, ${ }^{5}$ vomiting, and lifting during pregnancy. ${ }^{6,7}$ The prognosis for visual recovery is excellent as the haemorrhage resolves without compromising visual acuity. It is very important to rule out predisposing risk factors, including diabetes, sickle cell disease, anaemia, and other blood dyscrasias.

For most patients, only observation is required and the condition usually resolves within a few weeks to months. ${ }^{1}$ Further strenuous activity should be avoided until resolution of the haemorrhage. Large pre-retinal haemorrhages may require Nd:YAG membranotomy, which hastens recovery by allowing blood to gravitate through a laser-disrupted internal limiting membrane of the retina into the inferior vitreous cavity. ${ }^{8}$ Epiretinal membranes may form after Nd:YAG membranotomy. ${ }^{9}$ Pars plana vitrectomy may be required for long-standing large pre-retinal haemorrhages. For this patient, although the pre-retinal haemorrhage was large, it was decided to observe it with the resultant complete resolution after 7 months. Observation is considered an important management option, even for a large pre-retinal haemorrhage. This, of course, depends on the patient's visual status and wishes.

\section{References}

1. Chapman-Davis A, Lazarevic A. Valsalva maculopathy. Clin Exp Optom. 2002;85:42-5.

2. Duane TD. Valsalva hemorrhagic retinopathy. Trans Am Ophthalmol Soc. 1972;70:298-313.

3. Roberts DK, Mackay KA. Microhemorrhagic maculopathy associated with aerobic exercise. J Am Optom Assoc. 1987;58:415-8.

4. Georgiou T, Pearce I, Taylor RH. Valsalva retinopathy associated with blowing ballons. Eye. 1999;13:686-7.

5. Friberg TR, Braunstein RA, Bressler NM. Sudden visual loss associated with sexual activity. Arch Ophthalmol. 1995;113:738-42.

6. Callender D, Beirouty ZA, Saba SN. Valsalva hemorrhagic retinopathy in a pregnant woman. Eye. 1997;11:137-8.

7. Deane JS, Ziakas N. Valsalva retinopathy in pregnancy. Eye. 1997; 11;137-8.

8. Zaman F, Irwin R, Godley BF. Nd:YAG laser treatment for macular preretinal hemorrhage. Arch Ophthalmol. 1999;117:694-5.

9. Kwok AK, Lai TY, Chan NR. Epiretinal membrane formation with internal limiting membrane wrinkling after Nd:YAG laser membranotomy in Valsalva retinopathy. Am J Ophthalmol. 2003;136:763-6. 